\title{
Honey Bees Can Taste Amino and Fatty Acids in Pollen, but Not Sterols
}

\author{
Fabian A. Ruedenauer ${ }^{1 \dagger}$, Niklas W. Biewer ${ }^{2}$, Carmen A. Nebauer ${ }^{3}$, Maximilian Scheiner², \\ Johannes Spaethe ${ }^{3}$ and Sara D. Leonhardt ${ }^{1 * t}$
}

'Department of Life Science Systems, Research Department Life Science Systems, Technical University of Munich, Freising, Germany, ${ }^{2}$ Department of Animal Ecology and Tropical Biology, Biocenter, University of Würzburg, Würzburg, Germany,

${ }^{3}$ Department of Behavioral Physiology and Sociobiology, Biocenter, University of Würzburg, Würzburg, Germany

\section{OPEN ACCESS}

Edited by:

Isabel Marques,

University of Lisbon, Portugal

Reviewed by:

Sampat Ghosh,

Andong National University,

South Korea

Ramesh Sagill,

Oregon State University,

United States

*Correspondence:

Sara D. Leonhardt

leonhardt@wzw.tum.de

tORCID:

Fabian A. Ruedenauer orcid.org/0000-0002-2509-0554

Sara D. Leonhardt

orcid.org/0000-0002-8154-9569

Specialty section:

This article was submitted to Behavioral and Evolutionary Ecology,

a section of the journal

Frontiers in Ecology and Evolution

Received: 22 March 2021

Accepted: 31 May 2021

Published: 25 June 2021

Citation:

Ruedenauer FA, Biewer NW,

Nebauer CA, Scheiner M, Spaethe J and Leonhardt SD (2021) Honey Bees Can Taste Amino and Fatty Acids in Pollen, but Not Sterols.

Front. Ecol. Evol. 9:684175. doi: 10.3389/fevo.2021.684175
The nutritional composition of food is often complex as resources contain a plethora of different chemical compounds, some of them more, some less meaningful to consumers. Plant pollen, a major food source for bees, is of particular importance as it comprises nearly all macro- and micronutrients required by bees for successful development and reproduction. However, perceiving and evaluating all nutrients may be tedious and impair quick foraging decisions. It is therefore likely that nutrient perception is restricted to specific nutrients or nutrient groups. To better understand the role of taste in pollen quality assessment by bees we investigated nutrient perception in the Western honey bee, Apis mellifera. We tested if the bees were able to perceive concentration differences in amino acids, fatty acids, and sterols, three highly important nutrient groups in pollen, via antennal reception. By means of proboscis extension response (PER) experiments with chemotactile stimulation, we could show that honey bees can distinguish between pollen differing in amino and fatty acid concentration, but not in sterol concentration. Bees were also not able to perceive sterols when presented alone. Our finding suggests that assessment of pollen protein and lipid content is prioritized over sterol content.

Keywords: nutrient perception, proboscis extension response, plant-pollinator-interactions, resource use, gustation

\section{INTRODUCTION}

Like other animals, bees need to consume nutrients to maintain their homeostasis and produce progeny (Filipiak et al., 2017). Some nutrients (i.e., non-essential nutrients) can be synthesized by using components of other nutrients as building material. In contrast, essential nutrients cannot be synthesized and need to be ingested with food. Nutrients required in relatively high amounts are termed macronutrients, i.e., carbohydrates, protein, and fat, while micronutrients are required in relatively small amounts, i.e., trace minerals or vitamins (Simpson and Raubenheimer, 2012). Protein consists of amino acids, which are needed for the synthesis of endogenic proteins (Chapman, 1998) and for larval growth (DeGroot, 1953). They additionally provide energy to flight muscles (Micheu et al., 2000). Fat consists of fatty acids, which mostly provide and store energy, but also show antibiotic properties against several pathogens, like the American foulbrood causing agent Bacillus larvae (Feldlaufer et al., 1993), and they may enhance cognitive performance (Arien et al., 2015, 2018). Besides fatty acids, sterols represent particularly important lipids and are essential for many insects (Hobson, 1935; Svoboda et al., 1978), since they can act as messengers in the cellular membrane and as precursors for hormones such as the molting hormone (Svoboda et al., 1978). The performance and well-being of bees and other insects does depend on both the quality 
(i.e., chemical composition) and quantity (i.e., overall amount) of consumed nutrients. Several studies have shown that deviations from optimal nutrient ratios (Simpson and Raubenheimer, 2012) or over- and under-consumption of specific nutrients can lead to reduced survival and impair reproductive success in honey bees (Altaye et al., 2010; Arien et al., 2018) and bumble bees (Vaudo et al., 2016a,b; Moerman et al., 2017; Grund-Mueller et al., 2020; Ruedenauer et al., 2020).

Bees are unique in that they can obtain all essential and non-essential micro- and macronutrients from floral resources, i.e., mostly pollen and nectar (Haydak, 1970). Nectar is the main source of carbohydrates (i.e., sugars) and only contains low amounts of other nutrients (Baker, 1977; Nicolson and Thornburg, 2007). Pollen, in contrast, provides all other nutrients (DeGroot, 1953; Keller et al., 2005). However, pollen nutrient content can significantly differ within the same and among different plant species (Roulston and Cane, 2000; Roulston et al., 2000; Hanley et al., 2008; Ruedenauer et al., 2019b). As a consequence of such strong variation, bees need to assess the nutritional content of pollen when foraging to ensure an appropriate nutrient intake for themselves and their offspring and thus proper health and development. Nutritional quality assessment may take place directly through pollen nutrient perception at flowers or indirectly through physiological (e.g., nausea) or larval feedback (Behmer, 2009). Direct assessment requires the sense of taste using external chemoreception through chemotactile nutrient receptors, since nutrients are rarely volatile. In fact, bumble bees (Bombus terrestris) are able to perceive free amino and fatty acids (Ruedenauer et al., 2019a, 2020), whose concentrations correlate with their respective macronutrients (Ruedenauer et al., 2019b). Honey bees were found to use chemotactile cues to differentiate between pollen of different plant species, indicating that they may also use chemotactile cues to detect variations in nutrient composition (Ruedenauer et al., 2018). It is, however, still unknown, which pollen components honey bees can perceive and thus may use to assess pollen quality. Interestingly, we found that $B$. terrestris does "ignore" specific nutrients, e.g., amino acids, in pollen, even though they can perceive them when presented in isolation (Ruedenauer et al., 2019a). Instead they focused on (i.e., "prioritized" perception of) fatty acids in pollen (Ruedenauer et al., 2020). This finding indicates that bees restrict perception to specific nutrients when faced with a multitude of different compounds in their food resources.

To elucidate which nutrients can be perceived by the honey bee, Apis mellifera, we used a classical behavioral assay that conditions the proboscis extension response [PER, Bitterman et al. (1983), Matsumoto et al. (2012), Scheiner et al. (2013)]. PER conditioning makes use of the innate behavior of bees to extend their proboscis in response to sucrose stimulation (Bitterman et al., 1983), and has also been successfully adapted to test chemotactile stimuli like nutrients (Ruedenauer et al., 2015, 2019a, 2020). Differential conditioning of the PER can be used to test if bees are able to differentiate between different stimuli, e.g., different concentrations of the same nutrients. It therefore enables us to test if the bees are able to perceive concentration differences of a specific nutrient or nutrient group through manipulating their concentration, e.g., in pollen.

In this study we investigated whether honey bees are able to perceive concentration differences in amino acids, fatty acids and sterols (presented in isolation or in pollen) by means of chemotactile PER conditioning. Based on our previous results with bumble bees (Ruedenauer et al., 2020), which show similarities to honey bees in foraging behavior and social organization (Michener, 2000), we hypothesized that honey bees can only perceive concentration differences in pollen fatty acids and the structurally similar sterols, but ignore differences in amino acid concentrations, while they may be able to perceive all compound groups when presented in isolation.

\section{MATERIALS AND METHODS}

\section{Bee Colonies}

All experiments were performed with foragers of the western honey bee (Apis mellifera carnica) between June and August 2019 (pollen experiments) and in October 2020 (filter paper experiments). Honey bees were kept in Dadant bee hives at the Biocenter of the University of Würzburg, Germany. The landscape surrounding the hives comprised hedges, gardens, grassland, and orchards, which enabled the colonies to forage on a variety of different plant species (Kriesell et al., 2017). Therefore, colonies were healthy and of normal size. We tested bees from three different hives. In the late morning of sunny and warm days, we collected five departing foragers at the nest entrance of each colony, resulting in a total of fifteen bees tested per day. Bees of each colony were placed in separate containers. We did not differentiate between nectar and pollen foragers, as our aim was to obtain a general overview on nutrient perception in honey bees, though this might have increased overall variation in responses.

\section{Preparation of Stimuli}

We used a bee-collected pollen mix (Naturwaren-Niederrhein $\mathrm{GmbH}$, Goch-Asperden, Germany), which was ground in an electronic coffee grinder (CM 800, Graef, Arnsberg, Germany) to produce a powder which ensured homogenization of pollen from different plant species and thorough mixing with the added substances. The pollen mix contains pollen from about fifteen different genera and sustains healthy colony development in honey bees and bumble bees (Ruedenauer et al., 2016). Pollen stimuli were prepared as described in Ruedenauer et al. (2020). For each stimulus, we added $24 \mathrm{~g}$ of ground pollen into a petri dish. We then added ten times the natural concentrations of either eleven different amino acids (10x AA), seven fatty acids (10x FA) or five sterols (10x SP), mixed them well in a coffee grinder and added $13 \mathrm{ml}$ (for AA) or $24 \mathrm{ml}$ (for FA and SP) of de-ionized water (henceforth referred to as water) to create nutritionally enriched pollen pastes of similar consistencies (for details of the used AAs, FAs and SPs, see Supplementary Tables 1-3). Amino acids were selected to represent a spectrum of different amino acids typically found in pollen of flowers (Ruedenauer et al., 2019b) and representing both essential and 
non-essential ones for bees (according to DeGroot, 1953), as well as different chemical characteristics with regard to functional groups, polarity, and acidity. Moreover, these were the same amino acids as already used in a similar experiment with bumble bees (Ruedenauer et al., 2019a). The fatty acids used also corresponded to the ones used in previous experiments (Ruedenauer et al., 2020) and represent fatty acids typically found in pollen (Ruedenauer et al., 2019b). Unfortunately, many sterols, which are frequently found in pollen (Ruedenauer et al., 2019b) cannot be easily purchased; we therefore selected a spectrum of common pollen sterols that were commercially available. Average literature values of nutrient concentrations in pollen of a variety of different plant species were used as a reference to estimate natural concentrations (Manning, 2006; Weiner et al., 2010; Vanderplanck et al., 2014). We used ten times the average natural concentrations as they are still within the natural variation observed in pollen (Ruedenauer et al., 2019b) and found to be differentiated by Bombus terrestris in earlier studies (Ruedenauer et al., 2019a, 2020). To create a pure (non-nutritionally enriched) pollen paste, we only added water to the ground pollen. Pollen pastes were frozen at $-20^{\circ} \mathrm{C}$ and allowed to defrost for half an hour before usage.

We found that honey bees were not able to perceive concentration differences of sterols in pollen (see section "Results"). Such a lack of behavioral perception may due to the bees focusing on other substances (than sterols) in the chemically complex pollen mixture, while they may still perceive sterols when presented in isolation [as shown for amino acids in bumble bees, Ruedenauer et al. (2019a, 2020)]. Alternatively, they may not at all be able to perceive sterols. To differentiate between these two possibilities, we additionally tested if honey bees are able to perceive pure sterols, i.e., isolated from other compounds found in pollen. For this, we dissolved all sterols used in the pollen experiment in $1 \mathrm{ml}$ chloroform in their ten-fold natural concentration (10x SC, see Supplementary Table 3 for amounts of individuals sterols) to obtain the same concentrations as used in the pollen experiment. To prevent concentration changes due to solvent evaporation, the mixture was always prepared on ice directly before usage.

\section{Experimental Procedure}

The experimental procedure was based on Sommerlandt et al. (2014) and Ruedenauer et al. (2015). The bees were chilled on ice for $10 \mathrm{~min}$ in order to immobilize them, and were then harnessed in plastic tubes $(25 \mathrm{~mm} \times 10 \mathrm{~mm})$. Bees were fixed with a $1 \mathrm{~mm}$ crepe tape strip behind the head and a $10 \mathrm{~mm}$ strip wrapped around the tube to prevent movement except for antennae and proboscis. After $5 \mathrm{~min}$, the harnessed bees were fed $4 \mu \mathrm{l}$ of a $30 \%$ w/w sucrose solution with a micropipette and kept for $3 \mathrm{~h}$ in a climate chamber $\left(25^{\circ} \mathrm{C}, 60 \%\right.$ humidity, constant darkness $)$.

The experiments were conducted at constant temperature of $22^{\circ} \mathrm{C}$ and under daylight conditions complemented by artificial light. All experimenters wore gloves during the experiments.

After the $3 \mathrm{~h}$ starvation period, bees were tested for a proper PER by touching their antennae with a tooth pick, soaked in $30 \% \mathrm{w} / \mathrm{w}$ sucrose solution. Bees that responded with a PER (ca., $84 \%$ of all bees) were allowed to consume a small drop of sucrose solution. Bees not showing a PER were excluded from the experiment. For conditioning, each bee was placed in a rack and left resting for $15 \mathrm{~s}$. We then presented the nutrient stimulus (i.e., conditioned stimulus, CS: either pollen paste or dissolved sterol) on a copper plate [3 $\mathrm{mm} \times 4 \mathrm{~mm}$, Scheiner et al. (1999) and Ruedenauer et al. (2015)] by moving it toward the bee's left antenna. The bee was allowed to scan the stimulus for $6 \mathrm{~s}$ and we recorded if it showed a PER to the CS during the stimulation. Nutrient stimuli were prepared by placing $15 \mathrm{mg}$ of pollen paste on a $5 \mathrm{~mm} \times 5 \mathrm{~mm}$ wet piece of filter paper (6.8 $\mathrm{mg}$ after being soaked in water) or $5 \mu \mathrm{l}$ of sterol extract on dry filter paper. Filter papers with pollen paste were always prepared directly before the stimulation (to prevent drying), while filter papers with dissolved sterols were prepared $10 \mathrm{~min}$ before the experiment to allow for complete evaporation of the solvent. All plates were cleaned in 99\% ethanol (Hartenstein, Würzburg, Germany) after each stimulation. Three seconds after presenting the CS to the left antenna, the right antenna was touched with a wooden tooth pick. The tooth pick was either soaked in $50 \%$ sucrose solution (representing the unconditioned stimulus, US) as a reward (in CS+ trials) or blank (in CStrials). With this approach we could test whether the bees learn to differentiate between the rewarded $(\mathrm{CS}+)$ and unrewarded (CS-) stimulus. After stimulation, the bee was allowed to rest for $15 \mathrm{~s}$ before being replaced by the next bee. After $8 \mathrm{~min}$ the same individual was tested again [intertrial interval (ITI), Bitterman et al. (1983)]. We conducted 20 trials for each bee, ten CS+ and ten CS- presentations in a pseudorandomized order. When bees responded with a PER after stimulation with either $\mathrm{CS}+$ or CS-, it was scored as a positive response to the CS (i.e., scored as 1). When bees did not respond to either stimulus with a PER, but only showed a PER to the US (i.e., sugar water), it was scored as a negative response to the CS (i.e., scored as 0 ). Bees that did not respond to the US were scored with NA. If they did not respond for more than four times in a CS+ trial, they were excluded from the experiment. For bees scoring NA only up to a maximum of four times and then continued to show a PER upon CS, the NA responses were switched to no responses $(0)$ at the end.

When pollen paste was used as stimulus, we always tested the pollen paste enriched with nutrients (10x AA, 10x FA, or 10x SP) against pure pollen paste. Sterols dissolved in chloroform were tested against chloroform only. To control for the effect of stimulus type used as either CS+ or CS - , the same stimuli were always tested with reversed meanings, i.e., each stimulus was once tested as CS+ and once as CS- for two different sets of bees.

\section{Statistical Analyses}

To assess learning performance, we tested for differences in the positive PER responses to the stimulus between $\mathrm{CS}+$ and CS-. We used a binomial generalized additive mixed model (GAMM) to test for differences in responses between the two conditioned stimuli CS+ and CS - in relation to "stimulus type" (i.e., pure, 10x AA, 10x FA, 10x SP, or 10x SC). We used "trial" as smoother and "bee colony" and "bee individual" as random factors in the GAMM to take into account colony-specific variation and data dependency as each bee individual contributed with 20 data points (i.e., trials). Additionally, this approach also allowed us to analyze differences between stimulus types while 
taking into account variation induced by reversed meanings of the CS. Specifically, if bees showed differences in learning patterns depending on the type of stimulus presented as rewarded $(\mathrm{CS}+)$ and unrewarded $(\mathrm{CS}-)$, this would result in a significant interaction between CS and stimulus type. If the interaction was not significant, we merged the two datasets for the two reversed meanings. We did not find any significant interactions between conditioned stimulus and "stimulus type" (Supplementary Table 4). Therefore, data of both experimental series were merged for all datasets, following the standard procedure for PER conditioning experiments (Laloi et al., 1999; Sommerlandt et al., 2014).

If bees were able to differentiate between the different stimuli and thus different nutrient concentrations presented in the pollen paste (all nutrients) or chloroform (sterols), we considered them able to perceive/taste the tested nutrients. We additionally assessed differences in the number of trials required by bees to significantly differentiate between the two stimuli $(\mathrm{CS}+$ and CS-) using generalized linear mixed effect models (GLMM, with individual bee as random factor) for each trial. All statistical analyses were performed using R v4.0.3 (R Core Team, 2020).

\section{RESULTS}

Honey bees were able to learn the difference between pure pollen and 10x AA pollen $(F=4.398 ; P=0.036$, Figure 1$)$, as well as pure pollen and 10x FA pollen $(F=21.072, P<0.001$, Figure 2). However, PER responses differed significantly between the AA and FA experiment. When presented pure pollen and pollen enriched with FA, the bees significantly differentiated between $\mathrm{CS}+$ and CS - already from the fourth trial onward (GLMM: trial 1-3: ns, trial 4: $\chi^{2}=122.5, P<0.001$, Figure 2). However, when the bees were presented with pure pollen and pollen enriched with AA, discrimination was only significant from the sixth trial onward (GLMM: trial 1-5: ns, trial 6: $\chi^{2}=129.58, P=0.006$, Figure 1).

In contrast to $\mathrm{FA}$ and $\mathrm{AA}$, the bees were not able to discriminate between pollen differing in sterol concentrations, irrespective of whether the sterol stimulus was presented in pollen $(F=1.940, P=0.164$, Figure 3$)$ or chloroform $(F=2.179$, $P=0.140$, Figure 4).

\section{DISCUSSION}

The ability to perceive concentration differences of nutrients in pollen is a prerequisite for assessing the nutritional quality of different pollen sources. Our results suggest that honey bees can perceive and thus taste both amino (AAs) and fatty acids (FAs) but not sterols in pollen when using their antennae. The lack of perception of pollen sterols does not seem to be a consequence of selective perception of specific nutrients (e.g., AAs or FAs) in pollen, as indicated by the experiments with pure sterols. It rather hints at a general inability of $A$. mellifera to perceive this nutrient group via their antennae. Interestingly, these findings contradict our hypothesis that honey bees restrict nutrient perception in pollen to lipids, as has been shown for the bumble bee, Bombus

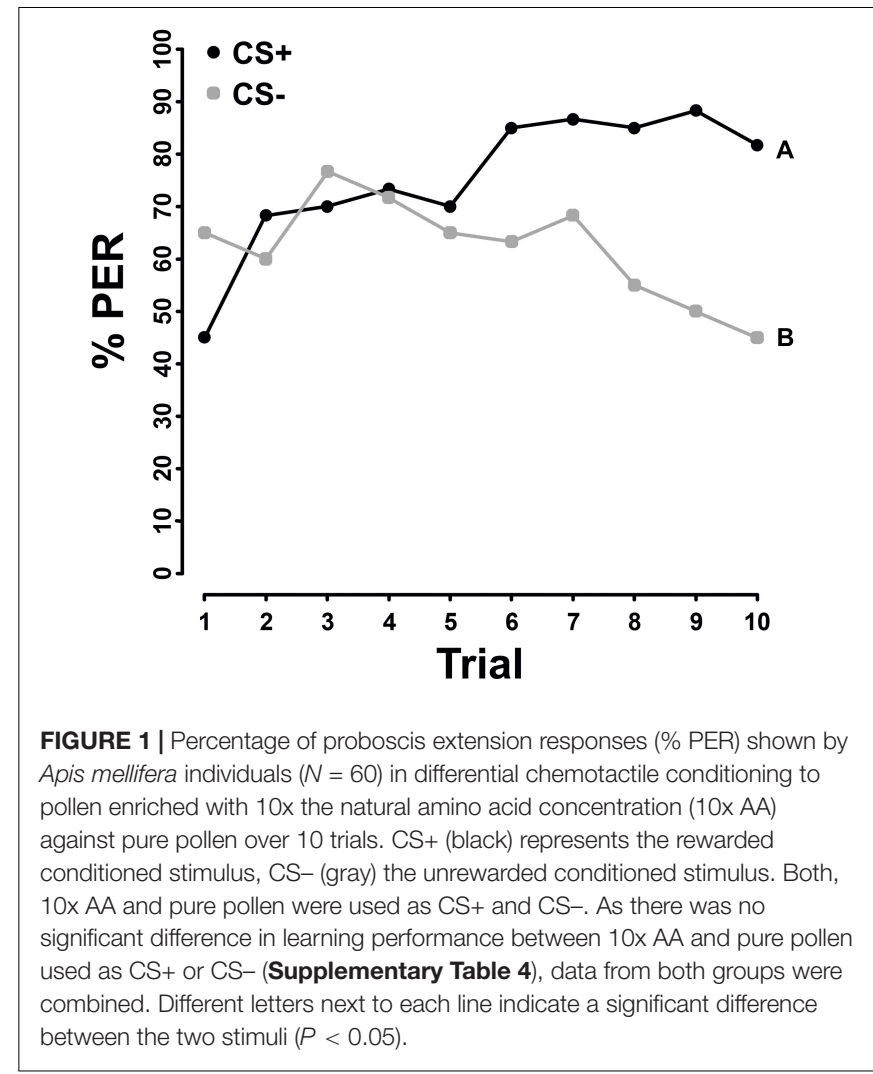

terrestris (Ruedenauer et al., 2020). While bumble bees can perceive AAs when presented in isolation (Ruedenauer et al., 2019a), they appear to "ignore" them and only respond to variation in FA content when part of a complex chemical mixture as represented by pollen (Ruedenauer et al., 2020).

The observed difference in perception of pollen nutrients between the two bee species may be related to species-specific differences in nutrient intake regulation. While bumble bees focus on the protein to lipid ratio (P:L-ratio) and specifically regulate fat intake (Vaudo et al., 2016a,b; Ruedenauer et al., 2020), honey bees seem to focus on the protein to carbohydrate ratio (P:C-ratio) and mainly regulate protein intake (Altaye et al., 2010; Pirk et al., 2010; Stabler et al., 2015), possibly in addition to the P:L ratio (Vaudo et al., 2020). The content of free AA in pollen correlates with its protein content (Ruedenauer et al., 2019b). Through assessing pollen AA and FA content, honey bees may consequently be able to regulate both protein and fat intake as well as their ratio.

Interestingly, the honey bees studied seemed to learn differences in pollen FA concentrations faster and more thoroughly than differences in pollen AA concentrations (see Figures 1 and 2). This finding suggests that it is easier for them to learn FA concentration differences than AA concentration differences, which might be related to the different effects that these two nutrient groups have on bee performance and thus fitness (Lepage and Boch, 1968; Vaudo et al., 2016b, 2020; Ruedenauer et al., 2020). For example, fat is detrimental to bees at much lower levels of overconsumption than protein (Canavoso et al., 2001; Harrison et al., 2012). It may therefore 


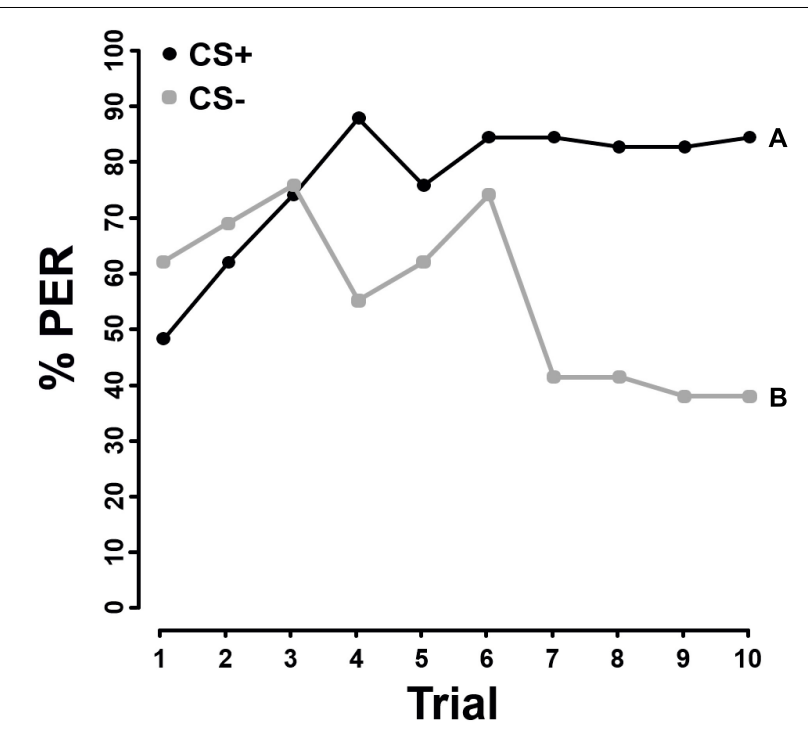

FIGURE 2 | Percentage of proboscis extension responses (\% PER) shown by Apis mellifera individuals $(N=58)$ in differential chemotactile conditioning to pollen enriched with $10 x$ the natural fatty acid concentration (10x FA) against pure pollen $(N=58)$ over 10 trials. $C S+$ (black) represents the rewarded conditioned stimulus, CS- (gray) the unrewarded conditioned stimulus. Both, 10x FA and pure pollen were used as CS+ and CS-. As there was no significant difference in learning performance between 10x FA and pure pollen used as CS+ or CS- (Supplementary Table 4), data from both groups were combined. Different letters next to each line indicate a significant difference between the two stimuli $(P<0.05)$.

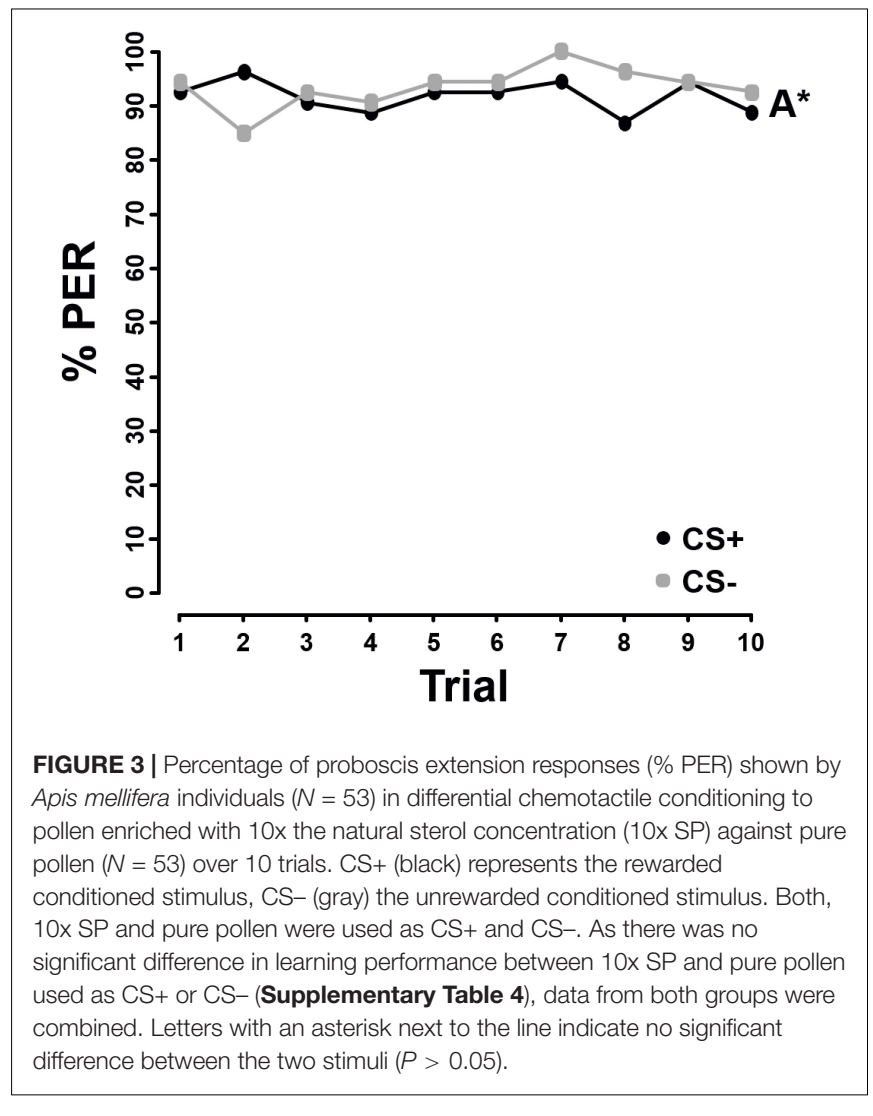

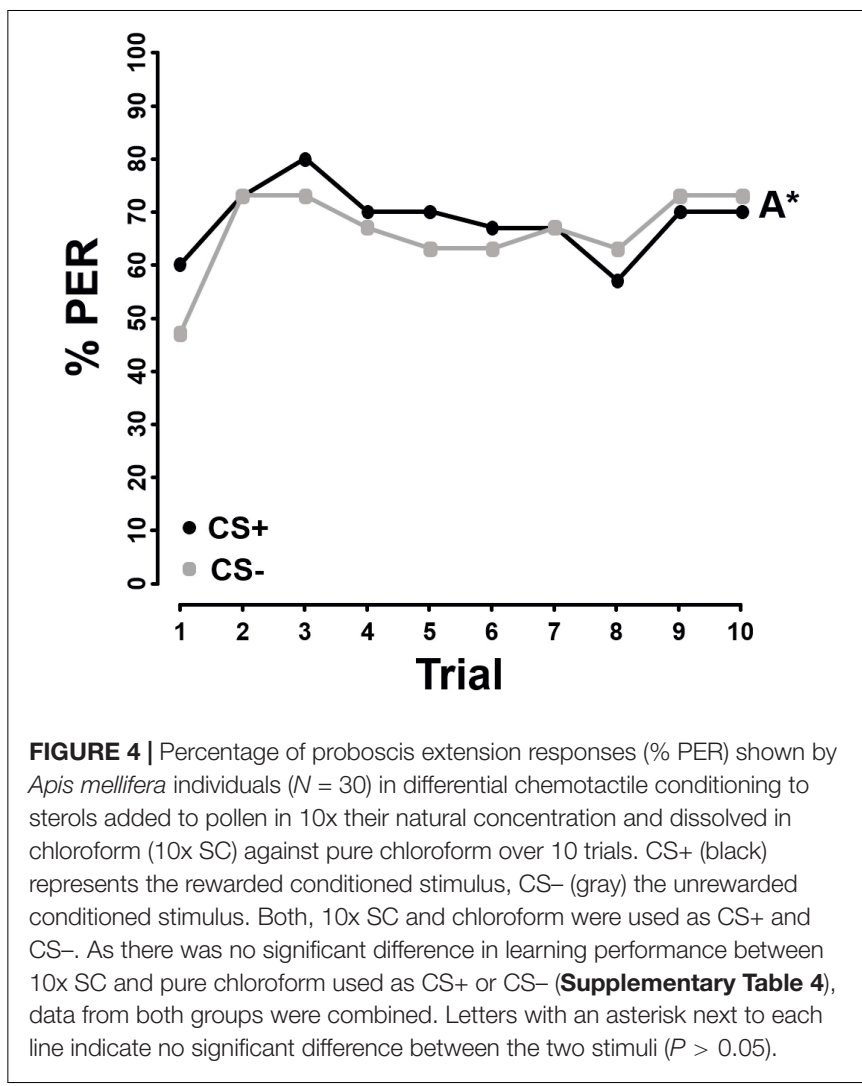

be adaptive for bees to be particularly sensitive to fat and strictly avoid its overconsumption. Such a link between nutrient perception and impact on animal fitness has recently also been suggested for Bombus terrestris (Ruedenauer et al., 2020).

Interestingly, and in contrast to our hypothesis, honey bees were not able to differentiate between different sterol concentrations or even to perceive pure sterols at all when using their antennae, suggesting that honey bees cannot receive sterols via their antennae. Given the importance of this nutrient group for bees in particular and insects in general (Hobson, 1935; Svoboda et al., 1978), this finding may at first seem surprising. However, sterol concentrations may simply be high enough in pollen of all or at least most plant species to fulfill the demand of honey bees, and/or variation in their concentrations as naturally found in pollen only barely impacts the bees' performance and reproduction. In fact, data from pollen analyzed so far (Vanderplanck et al., 2011; Somme et al., 2015; Roger et al., 2017; Ruedenauer et al., 2019b) shows that sterol contents in pollen vary less among different plant species than protein or fat contents. An alternative explanation for the lack of sterol perception in foragers might be that honey bee nurses alter the sterol composition of food when processing it inside the colony prior to provisioning their larvae. Unfortunately, precise information on the amounts and proportions of sterols required by bees and potential tolerances toward deviations from these are still unknown for the sterols used in our study (Herbert et al., 1980; Chakrabarti et al., 2019). 
We can, of course, not exclude that honey bees can perceive other sterols not included in our mixture, such as, e.g., 24methylene cholesterol, which seems to be highly important for honey bees (Vanderplanck et al., 2014; Chakrabarti et al., 2019). We can also not rule out that bees may perceive sterols by means other than their antennae, i.e., by tarsae or proboscis, or post-ingestively. The observed lack of learning may also be due to other reasons, such as aversiveness to the high concentrations of sterols used. In B. terrestris, however, internal, post-ingestive perception appears to complement external, preingestive perception (Ruedenauer et al., 2020), indicating that antennal perception may represent a reliable proxy for overall perception abilities. In fact, post-ingestive perception is mostly used to determine the body's current nutritional needs (Simpson and Raubenheimer, 1996). Pre-ingestive perception, in contrast, may therefore be especially important for polylectic bees, like honey bees and bumble bees, which need to obtain information on individual food/pollen sources collected before mixing and processing it for larval provisioning.

In conclusion, our study reveals bee species-specific differences in pre-ingestive antennal nutrient perception, which may be linked to species-specific differences in nutritional requirements, nutrient regulation and thus in the repertoire of chemical receptors or in the neuronal processing of chemical information. In fact, recent work found species-specific receptor gene expression for different bumble bee species (Sun et al., 2020), indicating that even closely related species that share many lifehistory traits may differ in their perceptive strategies, likely as a consequence of species-specific nutritional requirements.

\section{DATA AVAILABILITY STATEMENT}

The datasets presented in this study can be found in online repositories. The names of the repository/repositories and accession number(s) can be found below: https://osf.io/ w2xbv/?view_only=3713e9ca1fa44448889a33e240f43629 DOI: 10.17605/OSF.IO/W2XBV.

\section{REFERENCES}

Altaye, S. Z., Pirk, C. W. W., Crewe, R. M., and Nicolson, S. W. (2010). Convergence of carbohydrate-biased intake targets in caged worker honeybees fed different protein sources. J. Exp. Biol. 213, 3311-3318. doi: 10.1242/jeb. 046953

Arien, Y., Dag, A., and Shafir, S. (2018). Omega-6:3 ratio more than absolute lipid level in diet affects associative learning in honey bees. Front. Psychol. 9:1001. doi: 10.3389/fpsyg.2018.01001

Arien, Y., Dag, A., Zarchin, S., Masci, T., and Shafir, S. (2015). Omega-3 deficiency impairs honey bee learning. Proc. Natl. Acad. Sci. U.S.A. 112, 15761-15766. doi: $10.1073 /$ pnas. 1517375112

Baker, H. G. (1977). Non-sugar chemical constituents of nectar. Apidologie 8, 349-356. doi: 10.1051/apido:19770405

Behmer, S. T. (2009). Insect herbivore nutrient regulation. Annu. Rev. Entomol. 54, 165-187. doi: 10.1146/annurev.ento.54.110807.090537

Bitterman, M. E., Menzel, R., Fietz, A., and Schäfer, S. (1983). Classical conditioning of proboscis extension in honeybees (Apis mellifera). J. Comp. Psychol. 97, 107-119. doi: 10.1037/0735-7036.97.2.107

Canavoso, L. E., Jouni, Z. E., Karnas, K. J., Pennington, J. E., and Wells, M. A. (2001). Fat metabolism in insects. Annu. Rev. Nutr. 21, 23-46.

\section{AUTHOR CONTRIBUTIONS}

SL, JS, and FR conceived the experimental concepts. NB, CN, and MS performed the experiments. FR, NB, and MS analyzed the data. FR, SL, JS, and MS drafted the manuscript. All authors discussed the results, commented on the draft, and agreed to the final version.

\section{FUNDING}

Funding was provided by the Deutsche Forschungsgemeinschaft (DFG project: LE 2750/5-1/2 to SL and SP1380/1-1 and 1-2 to JS).

\section{ACKNOWLEDGMENTS}

We are very grateful for the constructive comments provided by the reviewers.

\section{SUPPLEMENTARY MATERIAL}

The Supplementary Material for this article can be found online at: https://www.frontiersin.org/articles/10.3389/fevo.2021. 684175/full\#supplementary-material

Supplementary Table 1 | Mean concentrations as naturally found in pollen and stimulus concentrations of amino acids (AA).

Supplementary Table 2 | Mean concentrations as naturally found in pollen and stimulus concentrations of fatty acids (FA).

Supplementary Table 3 | Mean concentrations as naturally found in pollen and stimulus concentrations of sterols (S).

Supplementary Table 4 | Statistical results of the generalized additive mixed effect models (GAMMs) testing for differences in learning performance between different stimuli.

Chakrabarti, P., Lucas, H. M., and Sagili, R. R. (2019). Evaluating effects of a critical micronutrient (24-Methylenecholesterol) on honey bee physiology. Ann. Entomol. Soc. Am. 113, 176-182. doi: 10.1093/aesa/saz067

Chapman, R. F. (1998). The Insects: Structure and Function. Cambridge: Cambridge university press.

DeGroot, A. P. (1953). Protein and amino acid requirements of the honey bee (Apis mellifica L.). Physiol. Comp. Oecol. 3, 197-285.

Feldlaufer, M. F., Knox, D., Lusby, W., and Shimanuki, H. (1993). Antimicrobial activity of fatty acids against Bacillus larvae, the causative agent of American foulbrood disease. Apidologie 24, 95-99. doi: 10.1051/apido:19930202

Filipiak, M., Kuszewska, K., Asselman, M., Denisow, B., Stawiarz, E., Woyciechowski, M., et al. (2017). Ecological stoichiometry of the honeybee: Pollen diversity and adequate species composition are needed to mitigate limitations imposed on the growth and development of bees by pollen quality. PLoS One 12:e0183236. doi: 10.1371/journal.pone.0183236

Grund-Mueller, N., Ruedenauer, F. A., Spaethe, J., and Leonhardt, S. D. (2020). Adding amino acids to a sucrose diet is not sufficient to support longevity of adult bumble bees. Insects 11:247. doi: 10.3390/insects11040247

Hanley, M. E., Franco, M., Pichon, S., Darvill, B., and Goulson, D. (2008). Breeding system, pollinator choice and variation in pollen quality in British herbaceous plants. Funct. Ecol. 22, 592-598. doi: 10.1111/j.1365-2435.2008.01415.x 
Harrison, J. F., Woods, H. A., and Roberts, S. P. (2012). Ecological and Environmental Physiology of Insects. Oxford: Oxford University Press.

Haydak, M. H. (1970). Honey bee nutrition. Annu. Rev. Entomol. 15, 143-156.

Herbert, E. W., Svoboda, J. A., Thompson, M. J., and Shimanuki, H. (1980). Sterol utilization in honey bees fed a synthetic diet: effects on brood rearing. J. Insect Physiol. 26, 287-289. doi: 10.1016/0022-1910(80)90135-3

Hobson, R. P. (1935). On a fat-soluble growth factor required by blow-fly larvae. II. Identity of the growth factor with cholesterol. Biochem J. 29, 2023-2026. doi: $10.1042 / \mathrm{bj} 0292023$

Keller, I., Fluri, P., and Imdorf, A. (2005). Pollen nutrition and colony development in honey bees: part 1. Bee World 86, 3-10. doi: 10.1080/0005772x.2005. 11099641

Kriesell, L., Hilpert, A., and Leonhardt, S. D. (2017). Different but the same: bumblebee species collect pollen of different plant sources but similar amino acid profiles. Apidologie 48, 102-116. doi: 10.1007/s13592-0160454-6

Laloi, D., Sandoz, J. C., Picard-Nizou, A. L., and Pham-Delegue, M. H. (1999). Olfactory conditioning of the proboscis extension reflex in the bumble bee Bombus terrestris. Ann. De La Soc. Entomol. France 35, 154-158.

Lepage, M., and Boch, R. (1968). Pollen lipids attractive to honeybees. Lipids 3, 530-534. doi: 10.1007/bf02530897

Manning, R. (2006). Fatty Acid Composition of Pollen and The Effect of Two Dominant Fatty Acids (Linoleic and Oleic) in Pollen and Flour Diets on Longevity and Nutritional Composition of Honey Bees (Apis mellifera). Ph.D. thesis. Perth, WA: Murdoch University.

Matsumoto, Y., Menzel, R., Sandoz, J. C., and Giurfa, M. (2012). Revisiting olfactory classical conditioning of the proboscis extension response in honey bees: a step toward standardized procedures. J. Neurosci. Methods 211, 159-167. doi: 10.1016/j.jneumeth.2012.08.018

Michener, C. D. (2000). The Bees of the World. Baltimore, MD: JHU Press.

Micheu, S., Crailsheim, K., and Leonhard, B. (2000). Importance of proline and other amino acids during honeybee flight. Amino Acids 18, 157-175. doi: 10.1007/s007260050014

Moerman, R., Vanderplanck, M., Fournier, D., Jacquemart, A.-L., and Michez, D. (2017). Pollen nutrients better explain bumblebee colony development than pollen diversity. Insect Conserv. Divers. 10, 171-179. doi: 10.1111/icad.12213

Nicolson, S. W., and Thornburg, R. W. (2007). "Nectar chemistry," in Nectaries and Nectar, eds S. W. Nicolson, M. Nepi, and E. Pacini (Berlin: Springer), 215-264. doi: 10.1007/978-1-4020-5937-7_5

Pirk, C. W. W., Boodhoo, C., Human, H., and Nicolson, S. (2010). The importance of protein type and protein to carbohydrate ratio for survival and ovarian activation of caged honeybees (Apis mellifera scutellata). Apidologie 41, 62-72. doi: $10.1051 /$ apido/2009055

R Core Team (2020). R: A Language and Environment for Statistical Computing. Vienna: R Foundation for Statistical Computing.

Roger, N., Michez, D., Wattiez, R., Sheridan, C., and Vanderplanck, M. (2017). Diet effects on bumblebee health. J. Insect Physiol. 96, 128-133. doi: 10.1016/j. jinsphys.2016.11.002

Roulston, T. A. H., Cane, J. H., and Buchmann, S. L. (2000). What governs protein content of pollen: pollinator preferences, pollen-pistil interactions, or phylogeny? Ecol. Monogr. 70, 617-643. doi: 10.1890/0012-9615(2000) 070[0617:wgpcop]2.0.co;2

Roulston, T. H., and Cane, J. H. (2000). Pollen nutritional content and digestibility for animals. Plant Syst. Evol. 222, 187-209. doi: 10.1007/978-3-7091-6306-1_10

Ruedenauer, F. A., Leonhardt, S. D., Lunau, K., and Spaethe, J. (2019a). Bumblebees are able to perceive amino acids via chemotactile antennal stimulation. J. Comp. Physiol. A 205, 321-331. doi: 10.1007/s00359-019-01321-9

Ruedenauer, F. A., Raubenheimer, D., Kessner-Beierlein, D., Grund-Mueller, N., Noack, L., Spaethe, J., et al. (2020). Best be(e) on low fat: linking nutrient perception, regulation and fitness. Ecol. Lett. 23, 545-554. doi: 10.1111/ele. 13454

Ruedenauer, F. A., Spaethe, J., and Leonhardt, S. D. (2015). How to know which food is good for you: bumblebees use taste to discriminate between different concentrations of food differing in nutrient content. J. Exp. Biol. 218, 22332240. doi: $10.1242 /$ jeb. 118554

Ruedenauer, F. A., Spaethe, J., and Leonhardt, S. D. (2016). Hungry for qualityindividual bumblebees forage flexibly to collect high-quality pollen. Behav. Ecol. Sociobiol. 70, 1209-1217. doi: 10.1007/s00265-016-2129-8
Ruedenauer, F. A., Spaethe, J., Van Der Kooi, C. J., and Leonhardt, S. D. (2019b). Pollinator or pedigree: which factors determine the evolution of pollen nutrients? Oecologia 191, 349-358. doi: 10.1007/s00442-019-04494-x

Ruedenauer, F. A., Wöhrle, C., Spaethe, J., and Leonhardt, S. D. (2018). Do honeybees (Apis mellifera) differentiate between different pollen types? PLoS One 13:e0205821. doi: 10.1371/journal.pone.0205821

Scheiner, R., Abramson, C. I., Brodschneider, R., Crailsheim, K., Farina, W. M., Fuchs, S., et al. (2013). Standard methods for behavioural studies of Apis mellifera. J. Apicult. Res. 52, 1-58. doi: 10.16984/saufenbilder.300635

Scheiner, R., Erber, J., and Page, R. E. (1999). Tactile learning and the individual evaluation of the reward in honey bees (Apis mellifera L.). J. Comp. Physiol. A Sens. Neural Behav. Physiol. 185, 1-10. doi: 10.1007/s003590050360

Simpson, S. J., and Raubenheimer, D. (1996). Feeding behaviour, sensory physiology and nutrient feedback: a unifying model. Entomol. Exp. Appl. 80, 55-64. doi: 10.1007/978-94-009-1720-0_13

Simpson, S. J., and Raubenheimer, D. (2012). The Nature of Nutrition: A Unifying Framework from Animal Adaptation to Human Obesity. Princeton: Princeton University Press.

Somme, L., Vanderplanck, M., Michez, D., Lombaerde, I., Moerman, R., Wathelet, B., et al. (2015). Pollen and nectar quality drive the major and minor floral choices of bumble bees. Apidologie 46, 92-106. doi: 10.1007/s13592-0140307-0

Sommerlandt, F. M. J., Rossler, W., and Spaethe, J. (2014). Elemental and nonelemental olfactory learning using PER conditioning in the bumblebee, Bombus terrestris. Apidologie 45, 106-115. doi: 10.1007/s13592-013-0227-4

Stabler, D., Paoli, P. P., Nicolson, S. W., and Wright, G. A. (2015). Nutrient balancing of the adult worker bumblebee (Bombus terrestris) depends on the dietary source of essential amino acids. J. Exp. Biol. 218, 793-802. doi: 10.1242/ jeb.114249

Sun, C., Huang, J., Wang, Y., Zhao, X., Su, L., Thomas, G. W. C., et al. (2020). Genus-wide characterization of bumblebee genomes provides insights into their evolution and variation in ecological and behavioral traits. Mol. Biol. Evol. 38, 486-501. doi: 10.1093/molbev/msaa240

Svoboda, J. A., Thompson, M. J., Robbins, W. E., and Kaplanis, J. N. (1978). Insect steroid metabolism. Lipids 13, 742-753. doi: 10.1007/bf02533755

Vanderplanck, M., Michez, D., Vancraenenbroeck, S., and Lognay, G. (2011). Micro-quantitative method for analysis of sterol levels in honeybees and their pollen loads. Anal. Lett. 44, 1807-1820. doi: 10.1080/00032719.2010.526271

Vanderplanck, M., Moerman, R., Rasmont, P., Lognay, G., Wathelet, B., Wattiez, R., et al. (2014). How does pollen chemistry impact development and feeding behaviour of polylectic bees? PLos One 9:e86209. doi: 10.1371/journal.pone. 0086209

Vaudo, A. D., Patch, H. M., Mortensen, D. A., Tooker, J. F., and Grozinger, C. M. (2016a). Macronutrient ratios in pollen shape bumble bee (Bombus impatiens) foraging strategies and floral preferences. Proc. Natl. Acad. Sci. U.S.A. 113, E4035-E4042.

Vaudo, A. D., Stabler, D., Patch, H. M., Tooker, J. F., Grozinger, C. M., and Wright, G. A. (2016b). Bumble bees regulate their intake of the essential protein and lipid pollen macronutrients. J. Exp. Biol. 219, 3962-3970.

Vaudo, A. D., Tooker, J. F., Patch, H. M., Biddinger, D. J., Coccia, M., Crone, M. K., et al. (2020). Pollen protein: lipid macronutrient ratios may guide broad patterns of bee species floral preferences. Insects 11:132. doi: 10.3390/ insects11020132

Weiner, C. N., Hilpert, A., Werner, M., Linsenmair, K. E., and Blüthgen, N. (2010). Pollen amino acids and flower specialisation in solitary bees. Apidologie 41, 476-487. doi: 10.1051/apido/2009083

Conflict of Interest: The authors declare that the research was conducted in the absence of any commercial or financial relationships that could be construed as a potential conflict of interest.

Copyright $\odot 2021$ Ruedenauer, Biewer, Nebauer, Scheiner, Spaethe and Leonhardt. This is an open-access article distributed under the terms of the Creative Commons Attribution License (CC BY). The use, distribution or reproduction in other forums is permitted, provided the original author(s) and the copyright owner(s) are credited and that the original publication in this journal is cited, in accordance with accepted academic practice. No use, distribution or reproduction is permitted which does not comply with these terms. 